\title{
The bacterium Wolbachia exploits host innate immunity to establish a symbiotic relationship with the dengue vector mosquito Aedes aegypti
}

\author{
Xiaoling Pan ${ }^{1,2}$, Andrew Pike ${ }^{1}$, Deepak Joshi ${ }^{1}$, Guowu Bian ${ }^{1}$, Michael J McFadden ${ }^{1}$, \\ Peng Lu ${ }^{1}$, Xiao Liang ${ }^{1}$, Fengrui Zhang ${ }^{1}$, Alexander S Raikhel ${ }^{3}$ and Zhiyong Xi ${ }^{1,4}$ \\ ${ }^{1}$ Department of Microbiology and Molecular Genetics, Michigan State University, East Lansing, MI 48824, \\ USA; ${ }^{2}$ School of Medicine, Hunan Normal University, Changsha, Hunan 410013, China; ${ }^{3}$ Department of \\ Entomology and Institute for Integrative Molecular Biology, University of California, Riverside, CA 92521, \\ USA and ${ }^{4}$ Sun Yat-sen University-Michigan State University Joint Center of Vector Control for Tropical \\ Diseases, Guangzhou, Guangdong 510080, China
}

\begin{abstract}
A host's immune system plays a central role in shaping the composition of the microbiota and, in return, resident microbes influence immune responses. Symbiotic associations of the maternally transmitted bacterium Wolbachia occur with a wide range of arthropods. It is, however, absent from the dengue and Zika vector mosquito Aedes aegypti in nature. When Wolbachia is artificially forced to form symbiosis with this new mosquito host, it boosts the basal immune response and enhances the mosquito's resistance to pathogens, including dengue, Zika virus and malaria parasites. The mechanisms involved in establishing a symbiotic relationship between Wolbachia and A. aegypti, and the long-term outcomes of this interaction, are not well understood. Here, we have demonstrated that both the immune deficiency (IMD) and Toll pathways are activated by the Wolbachia strain wAlbB upon its introduction into $A$. aegypti. Silencing the Toll and IMD pathways via RNA interference reduces the wAlbB load. Notably, wAlbB induces peptidoglycan recognition protein (PGRP)-LE expression in the carcass of $\boldsymbol{A}$. aegypti, and its silencing results in a reduction of symbiont load. Using transgenic mosquitoes with stage-specific induction of the IMD and Toll pathways, we have shown that elevated wAlbB infection in these mosquitoes is maintained via maternal transmission. These results indicate that host innate immunity is utilized to establish and promote host-microbial symbiosis. Our results will facilitate a long-term projection of the stability of the Wolbachia-A. aegypti mosquito system that is being developed to control dengue and Zika virus transmission to humans. The ISME Journal (2018) 12, 277-288; doi:10.1038/ismej.2017.174; published online 3 November 2017
\end{abstract}

\section{Introduction}

Mosquitoes transmit numerous devastating human diseases such as Zika, dengue and malaria. Both the Zika and dengue viruses are vectored primarily by the Aedes aegypti mosquito. While dengue fever has spread to five continents with over 3 billion people at risk of contracting the disease (Bhatt et al., 2013), Zika transmission recently resulted in a public health emergency of international concern. Failing mosquito-control methods, as well as the lack of an anti-dengue vaccine and anti-viral drugs, have contributed to this situation. Recently, several nonchemical insecticide-based approaches have been developed for the control of mosquito populations,

Correspondence: Z Xi, Department of Microbiology and Molecular Genetics, Michigan State University, 293 Farm Lane, Room 314, Giltner Hall, East Lansing, MI, 48824, USA.

E-mail: xizy@msu.edu

Received 7 June 2017; revised 30 August 2017; accepted 6 September 2017; published online 3 November 2017 including the utilization of Wolbachia (Xi et al., 2005; Bian et al., 2010; McMeniman and O'Neill, 2010; Hoffmann et al., 2011).

Wolbachia spp. are obligate, maternally inherited endosymbionts that infect $>65 \%$ of all the insect species and $\sim 28 \%$ of the surveyed mosquito species (Kittayapong et al., 2000; Ricci et al., 2002; Werren et al., 2008). Through cytoplasmic incompatibility (CI), Wolbachia induce early embryonic death when a Wolbachia-infected male mates with a female that is either uninfected or infected by a different type of Wolbachia (Dobson, 2003). Cytoplasmic incompatibility provides a reproductive advantage to infected females over uninfected females, resulting in the invasion of Wolbachia into a population. Wolbachia can also interfere with pathogen infection and directly inhibit a variety of human pathogens including dengue virus, Zika virus, malaria parasites and filarial worms (Kambris et al., 2009; Moreira et al., 2009; Bian et al., 2010, 2013; Dutra et al., 2016). These features of Wolbachia have generated a 
great deal of interest in creating Wolbachia-based systems for diminishing the mosquito's ability to transmit pathogens and/or suppress mosquito population (Xi et al., 2005; Brelsfoard et al., 2008; Hoffmann et al., 2011; Bian et al., 2013; Bourtzis et al., 2014). However, the mechanisms responsible for establishing persistent symbiosis between this bacterium and new hosts have not been well understood, despite the importance of this question for predicting the long-term success of Wolbachia-based vector-borne disease control programs.

Insects, including mosquitoes, have developed a highly effective innate immune system to defend against harmful microbes they encounter during their life cycle. The immune response is initiated by identification of microbe-associated molecular patterns via pattern-recognition receptors (PRRs), which results in the activation of immune signaling pathways and expression of effector molecules to suppress infection (Dimopoulos, 2003; Waterhouse et al., 2007). In mosquitoes, the Toll and immune deficiency (IMD) signaling pathways activate two distinct nuclear factor-kappaB-IkB transcription factors, REL1 and REL2, respectively, to induce the expression of antimicrobial peptides (Waterhouse et al., 2007). This innate immunity has a significant role in determining the mosquito's ability to transmit human pathogens, such as dengue virus and malaria parasites (Meister et al., 2005; Dong et al., 2006; Frolet et al., 2006; Xi et al., 2008b). While the Toll pathway functions to defend against Gram-positive bacteria, fungi, dengue virus and Plasmodium berghei (Bian et al., 2005; Shin et al., 2005; Frolet et al., 2006; Xi et al., 2008b), activation of the IMD pathway inhibits Gram-negative bacterium and Plasmodium falciparum (Meister et al., 2005). Overall, such responses to pathogen invasion characterize inducible immunity. In contrast, constitutive immunity, also referred to as basal immunity, represents a surveillance mechanism that functions prior to pathogen invasion. Data have shown that basal immunity is influenced by the commensal microbiota within a mosquito (Frolet et al., 2006). Removal of these resident microbes reduces the baseline level of immune effectors, resulting in increased susceptibility of the mosquito to both dengue virus and malaria parasites (Xi et al., 2008b; Dong et al., 2009). Recent studies have demonstrated that the symbiotic microbiota contributes to the buildup of host basal immunity and the function of certain immune responses in both vertebrates and invertebrates (Kumar et al., 2010; Hooper et al., 2012). For example, in multiple hosts, gut bacterial ligands from the microbiota signal through PRRs to promote the development of host tissues, including the immune system, and protect the host from diseases (Chu and Mazmanian, 2013).

Although A. aegypti, the primary dengue vector, does not carry Wolbachia in nature, the Wolbachia wAlbB strain has been successfully transferred from its native host, Aedes albopictus, into this mosquito species and, since then, the wAlbB-infected A. aegypti strain (WB1) has been maintained for $>12$ years in a laboratory population (Xi et al., 2005). In contrast to $A$. albopictus, in which no impact of Wolbachia on immunity has been observed (Bourtzis et al., 2000; Molloy and Sinkins, 2015), wAlbB induces the production of reactive oxygen species and activates the Toll pathway in WB1 strain A. aegypti (Pan et al., 2012). In addition, wAlbB has been shown to grow to significantly higher densities in WB1 than in A. albopictus and invade different tissues such as the fat body, midgut, salivary glands, and ovaries (Lu et al., 2012). In A. albopictus, the majority of Wolbachia reside in the ovaries (Lu et al., 2012). Although it is known that Wolbachia infection results in immune activation in transfected A. aegypti lines (Kambris et al., 2009; Moreira et al., 2009; Bian et al., 2010, 2013), it is unclear what role these immune responses have in establishing the symbiotic relationship of this bacterium and its new host. Specifically, it is unknown whether boosting the immune response prevents overproliferation of Wolbachia or promotes the establishment and maintenance of symbiosis with this bacterium. Elucidating this information is essential for predicting the long-term persistence of the symbiotic relationship of Wolbachia with its new host, A. aegypti, and the potential success of the Wolbachia-mosquito system that is presently being used to control mosquito populations.

In this work, we utilized reverse genetic and transgenic tools to manipulate the mosquito innate immune system and test its influence on the interaction between wAlbB and A. aegypti. Suppression of either the IMD pathway alone or both the Toll and IMD pathways reduced the Wolbachia load, whereas activation of both pathways increased Wolbachia load, indicating the importance of innate immunity in fostering the symbiotic relationship between $w$ AlbB and $A$. aegypti. Using transgenic mosquitoes with stage-specific induction of the IMD and Toll pathways, we have shown that the elevated wAlbB infection in this background is maintained via maternal transmission, which is a hallmark of an established symbiotic relationship. Overall, our results suggest that Wolbachia utilizes the host immune system to promote its symbiotic relationship with $A$. aegypti mosquitoes.

\section{Results}

Wolbachia wAlbB activates both the Toll and IMD pathways and protects A. aegypti against bacteria and fungi

We have previously shown that wAlbB infection activates the Toll pathway in A. aegypti (Pan et al., 2012). To determine a possible effect of wAlbB on the IMD pathway, we conducted quantitative reverse-transcription polymerase chain reaction (qRT-PCR) assays comparing the transcript 

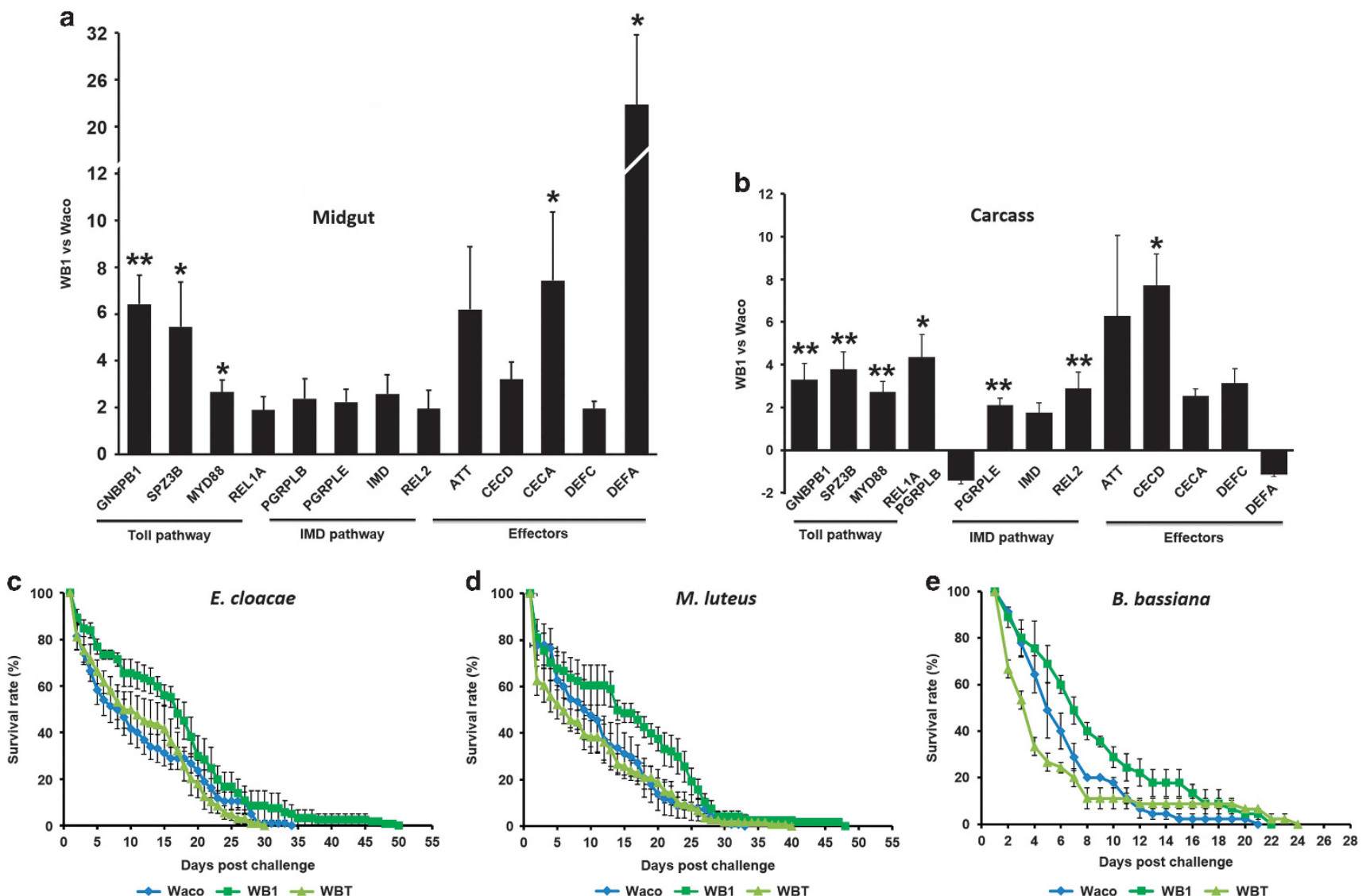

Figure 1 wAlbB induces both the IMD and Toll pathways in the A. aegypti line WB1. The fold change in the expression of Toll and IMD pathway genes, measured by qRT-PCR, in the midgut (a) and remaining carcass (b) of wAlbB-infected WB1 mosquitoes relative to the uninfected Waco line at 7 days old, prior to blood feeding. Each treatment had four biological replicates, with ten midguts or carcasses pooled as one sample. $2^{-\triangle \Delta \mathrm{CT}}$ method was used to calculate the fold change for each gene. Significance was determined based on comparison of the $\Delta \mathrm{CT}$ of each gene in WB1 and Waco using Student's $t$-tests. ${ }^{*} P<0.05 ;{ }^{*} P<0.01$. Survival curves of the mosquitoes post-challenge with E. cloacae (c), M. luteus (d) or B. bassiana (e). WB1 is an A. aegypti line carrying a stable wAlbB infection. WBT is an aposymbiotic line generated by removing wAlbB from WB1 by tetracycline treatment, and Waco is a wild-type Wolbachia-uninfected line. Each treatment had either six (c and d) or three (e) biological replicates of 15-20 mosquitoes each. Error bars indicate the standard error. Survival curves are significantly different between WB1 and other groups (compared using log-rank test).

abundance of 13 immune genes from the IMD and Toll pathways. Total RNA was prepared from midguts and the remaining carcass tissues of 7-dayold female mosquitoes of the WB1 and wild-type Waco strains. Consistent with the previous report (Pan et al., 2012), transcripts of three assayed Toll pathway genes-GNBPB1, SPZ3B and MYD88were strongly induced in both the midguts (6.4-, 5.5- and 2.7-fold, respectively; $P<0.05)$ and carcass tissues (3.3-, 3.8- and 2.7-fold, respectively; $P<0.01$ ) (Figures 1a and b). In addition, REL1 was induced 4.3 -fold by wAlbB in the carcass $(P<0.05)$ (Figure 1b). The transcripts of two assayed IMD pathway genes-peptidoglycan recognition protein (PGRP)-LE, and REL2-were elevated greater than twofold in the carcasses of WB1 when compared with Waco mosquitoes $(P<0.01)$ (Figure 1b); in the midguts of WB1, PGRP-LE, PGRP-LB, IMD and REL2 were induced about 2-fold, although not significantly. DEFA was induced $>22$-fold in the midgut $(P<0.05)$ of WB1 mosquitoes, and CECA and CECD were induced 7.4-fold and 7.7-fold in midguts and carcass $(P<0.05)$, respectively (Figures 1a and b). These results suggest that the simultaneous induction of Toll and IMD in WB1 mosquitoes may result in an enhanced synergistic effect on the expression of effector molecules, which is consistent with our previous report (Zou et al., 2011).

To test whether the wAlbB-based immunity boost in female mosquitoes affects the susceptibility of these mosquitoes to pathogens, we compared their survivorship after challenge with Gram-negative bacteria (Enterobacter cloacae), Gram-positive bacteria (Micrococcus luteus) or fungi (Beauveria bassiana) with that of the Waco wild-type strain and aposymbiotic WBT strain (generated by tetracycline treatment of WB1 mosquitoes to remove wAlbB). Consistent with IMD pathway induction, WB1 mosquitoes survived significantly better than both Waco $(P<0.01)$ and WBT $(P<0.001)$ after challenge with E. cloacae (Figure 1c). Activation of the Toll pathway is expected to protect mosquitoes from Gram-positive bacterial and fungal infections. Indeed, WB1 mosquitoes had a higher survival 

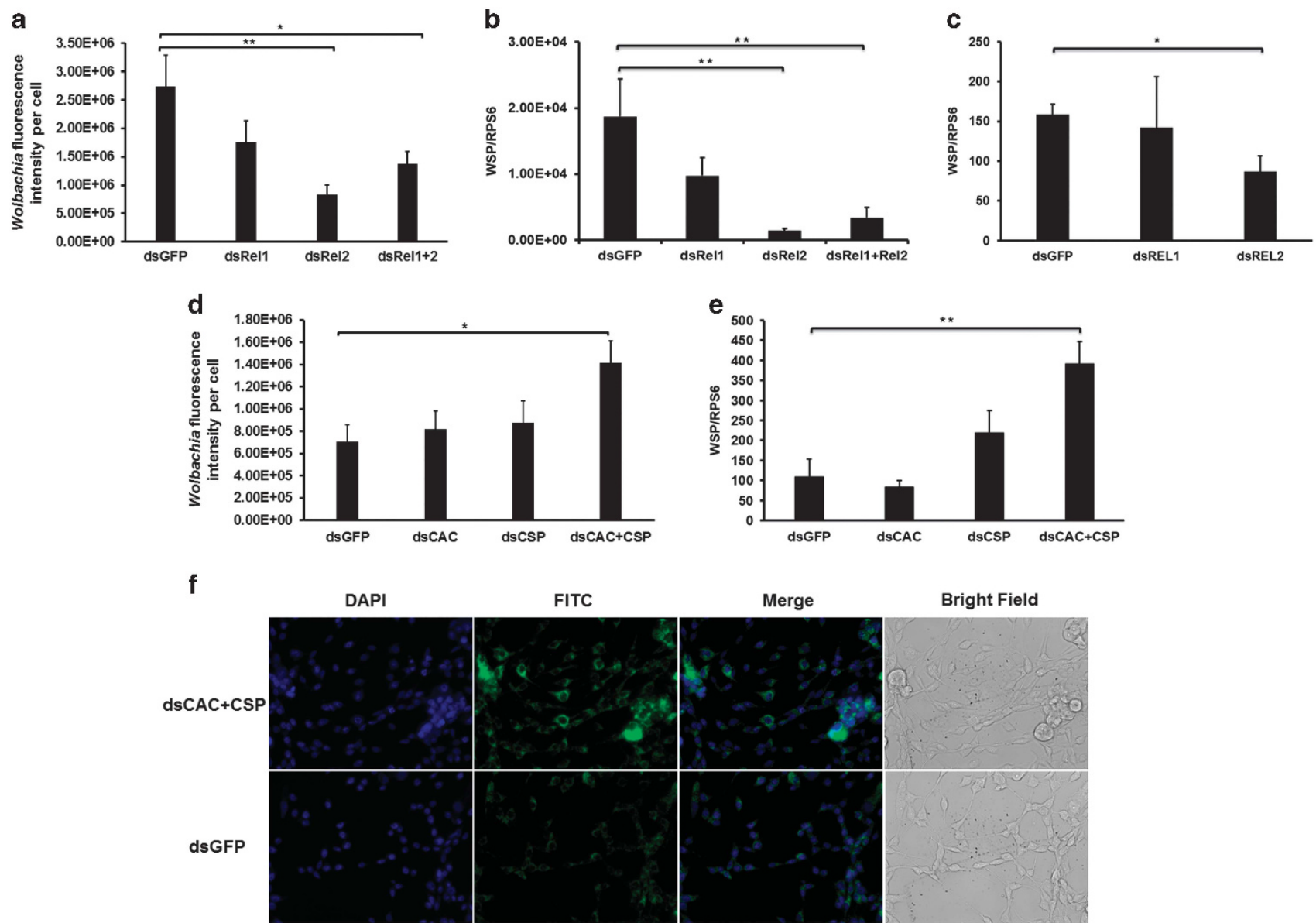

Bright Field
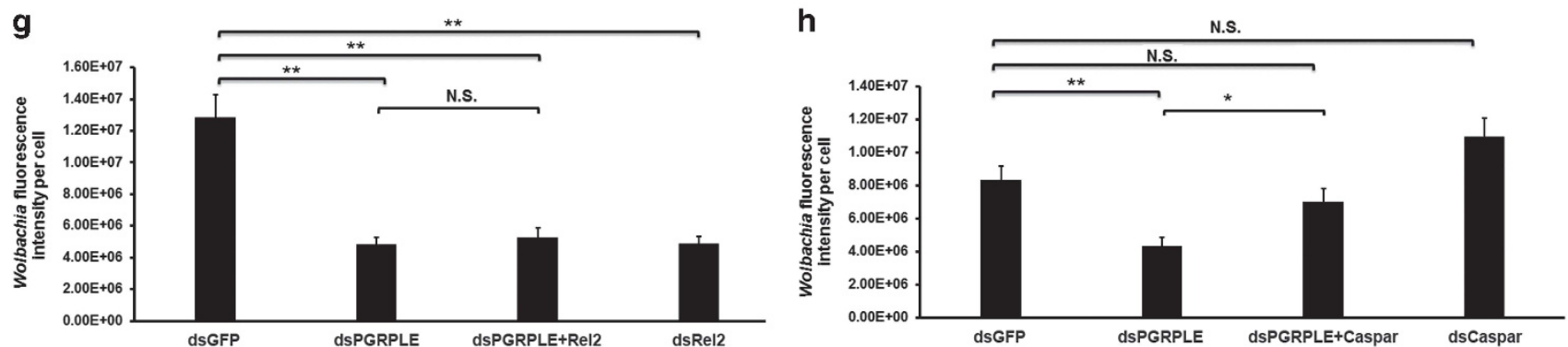

Figure 2 wAlbB load changes in response to manipulation of the Toll and IMD pathways via RNAi-mediated gene silencing. The amount of wAlbB was measured using either IFA (a) or real-time PCR (b and c) in the A. aegypti cell W-Aag2 (a and b) and WB1 mosquitoes (c) after treatment with dsRNA of Rel1, Rel2, both or GFP (control). The same measurements were performed again, after the negative regulators of the Toll and IMD pathways, cactus (CAC) and caspar (CSP), respectively, had been silenced either individually or together (CAC+CSP) to activate these pathways in the W-Aag2 cell line (d and e). Representative IFA images of dsRNA-treated W-Aag2 cells at x20 magnification with Wolbachia surface protein (WSP) staining (green) (f). The Wolbachia fluorescence intensity was measured in W-Aag2 cells after PGRP-LE was knocked down, either individually or together with either REL2 (g) or Caspar (h). Error bars indicate the standard error. ${ }^{*} P<0.05 ;{ }^{*} P<0.01$; N.S., no significance; one-way ANOVA.

rate than Waco and WBT when challenged with either M. luteus $(P<0.001)$ or $B$. bassiana $(P<0.05)$ (Figures $1 \mathrm{~d}$ and e).

Suppression of the IMD pathway reduces the levels of $\mathrm{wAlbB}$ in both A. aegypti cells and mosquitoes Because Wolbachia is a Gram-negative bacterium, we initially hypothesized that the boosted immunity, in particular the activation of the IMD pathway, might play an important role in preventing the over- proliferation of wAlbB in WB1 mosquitoes. To test this suggestion, we knocked down REL1, REL2 or both simultaneously using their respective doublestranded (ds) RNAs in the wAlbB-infected A. aegypti Aag2 cell line (W-Aag2). Wolbachia fluorescence intensity was measured using indirect IFA 3 days post treatment. Surprisingly, the level of wAlbB was reduced significantly in cells with either single REL2 silencing or a double knockdown of REL1 and REL2 when compared with the dsGFP-treated control (Figure 2a, Supplementary Figures S1A and B). The 
experiment was repeated independently, with the density of wAlbB measured using RT-PCR assay 5 days post treatment (Figure 2b). Consistently, the copy number of the wAlbB genome measured by taking the value of Wolbachia surface protein gene normalized to the host RPS6 gene was reduced 13.2and 5.6-fold after single REL2 and dual REL1/REL2 knockdowns in W-Aag2 cells, respectively (Figure 2b). To exclude the possibility that such reduction was caused by a change in the number of copies of host RPS6, we compared the absolute number of copies of Wolbachia surface protein among different treatments. With the silencing of REL2 and co-silencing of both REL1 and REL2, the Wolbachia surface protein copy number was significantly lower in W-Aag2 cells than in the control $(P<0.05)$ (Supplementary Figure S2). To further confirm this result, we carried out a similar knockdown in WB1 mosquitoes and measured the amount of Wolbachia in mosquito ovaries 4 days after treatment. Again, we observed a significant reduction in the wAlbB load in WB1 ovaries when REL2 was silenced (Figure 2c). We failed to get results from mosquitoes with the double knockdown of REL1 and REL2 because of a very high mortality in treated mosquitoes. These results indicate that suppression of the IMD pathway reduces Wolbachia density in both A. aegypti cells and mosquitoes.

Boosting mosquito immunity increases wAlbB load in A. aegypti cells

To test whether boosting the Toll and IMD pathways would produce an effect on Wolbachia opposite to that observed following suppression of these two pathways, we treated W-Aag2 cells with dsRNA of Cactus and Caspar, the respective negative regulators of the Toll and IMD pathways, to induce the individual pathway (Xi et al., 2008b). Double silencing of both Cactus and Caspar was also conducted to activate both pathways simultaneously. In two independent assays, wAlbB density was measured using either IFA or RT-PCR assay 3 or 5 days post treatment, respectively. The results consistently show a significant increase in the Wolbachia intensity in the cells following simultaneous silencing of Cactus and Caspar when compared with the dsGFP-treated control (Figures 2d-f; Supplementary Figures S3A and B). This increase was not observed in individual knockdowns of either Cactus or Caspar. These results show that boosting immunity promotes proliferation of wAlbB in mosquito cells.

The IMD pathway intracellular receptor PGRP-LE facilitates wAlbB growth in A. aegypti cells PGRP-LE has been shown to function upstream of the IMD pathway, serving as the only known intracellular PRR of Gram-negative bacteria in Drosophila (Kaneko et al., 2006; Waterhouse et al.,
2007; Yano et al., 2008). Because the expression of PGRP-LE was induced by wAlbB in the carcass of $A$. aegypti (Figure 1b), we investigated whether PGRPLE might have a role in sensing Wolbachia in the IMD pathway to facilitate wAlbB growth in A. aegypti. We conducted RNAi silencing of PGRPLE, REL2 and both, and measured their impact on wAlbB density in the W-Aag2 cell relative to that of the control group treated with dsGFP. We observed that Wolbachia fluorescent intensity was significantly reduced by 2.6-fold after either PGRP-LE or REL2 was silenced $(P<0.0001)$ (Figure 2g, Supplementary Figures S4A and B). Suppression of the IMD pathway by co-silencing both PGRP-LE and REL2 genes caused no further decrease of Wolbachia fluorescent intensity compared with the silencing of PGRP-LE alone (Figure 2g). To validate whether the effect of PGRP-LE on wAlbB infection level was modulated through the IMD pathway, we further compared wAlbB density in W-Aag2 cells after silencing PGRP-LE, Caspar, and both in W-Aag2 cells (Supplementary Figures S4C and D). We observed that, although the wAlbB density was reduced after silencing of PGRP-LE, it was restored to a level similar to the control group after double knockdown of PGRP-LE and Caspar (Figure 2h). These results indicate that both PGRP-LE and REL2 influence wAlbB through the IMD pathway, and PGRP-LE may serve as a PRR to sense Wolbachia upstream of the IMD pathway.

Boosting mosquito immunity increases $\mathrm{w} A l b B$ load in transinfected A. aegypti

Manipulating the immune pathway by mean of dsRNA-mediating gene silencing produces only a transient effect. To further test the effect of boosting the Toll and IMD pathways on Wolbachia in A. aegypti, we took advantage of transgenic lines that overexpress REL1 (REL1+) and REL2 (REL2+), and in which these immune regulatory factors are activated via the blood-meal inducible Vitellogenin (Vg) promoter (Bian et al., 2005; Antonova et al., 2009). wAlbB was introduced into REL1+ and REL2+ mosquito lines through repeated crosses of wAlbBinfected females with REL1+ and REL2+ transgenic males for seven generations (Bian et al., 2005; Antonova et al., 2009). These crosses resulted in $w$ AlbB-infected transgenic mosquitoes, referred to as $\mathrm{W}+/$ REL1+ and W+/REL2+. A cross of wAbBinfected mosquitoes with wild-type UGAL/Rockefeller strain mosquito, from which the transgenic lines were derived, was used to generate a control line, W+/UGAL. A single cross between W+/REL1 and $\mathrm{W}+/$ REL2+ was used to generate a hybrid line with co-activated Toll and IMD pathways, referred to as W+/REL1+/REL2+ (Figure 3a). To validate the activation of the transgenes in these lines, we measured the expression of REL1 and REL2 in mosquito fat bodies 7 days post eclosion and $24 \mathrm{~h}$ post blood meal (PBM). Consistent with previous 
282

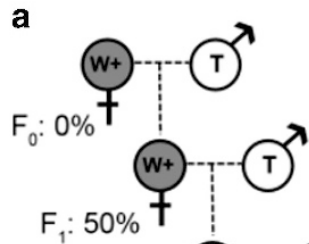

a
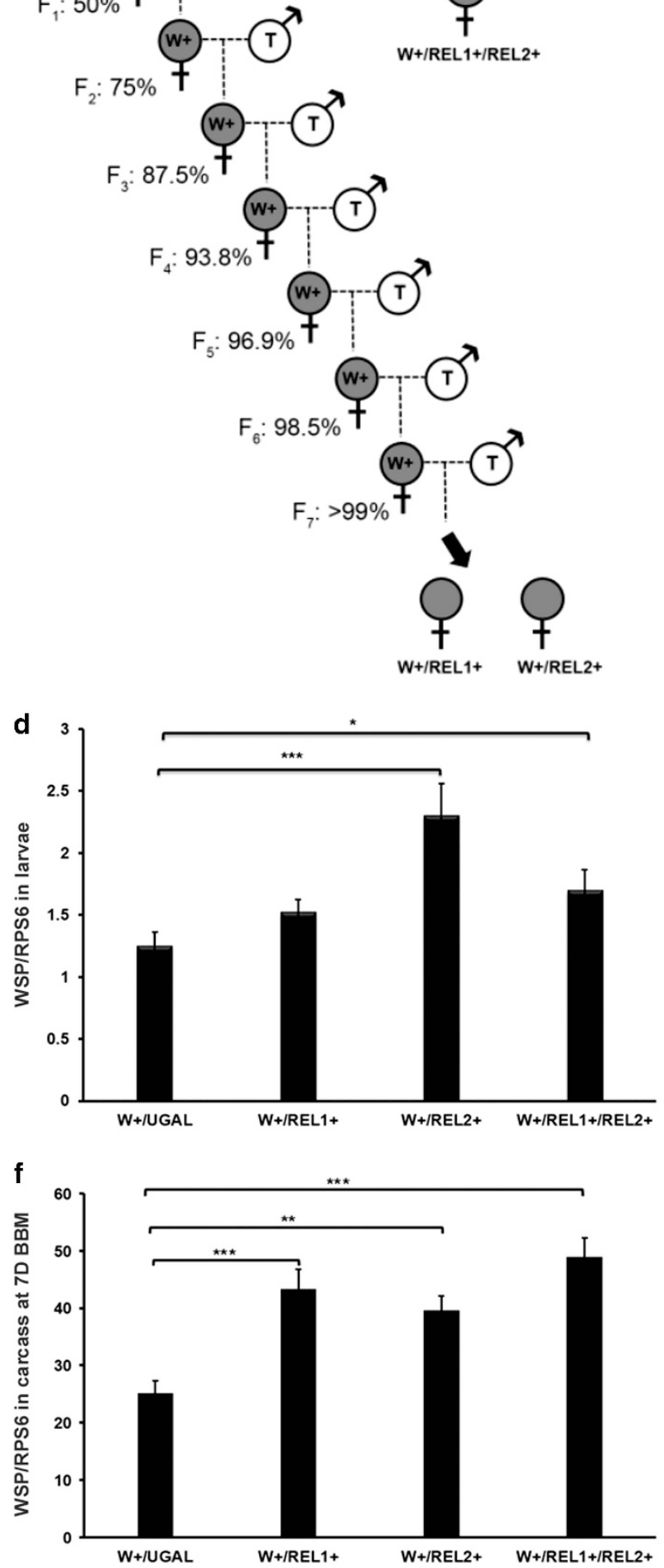
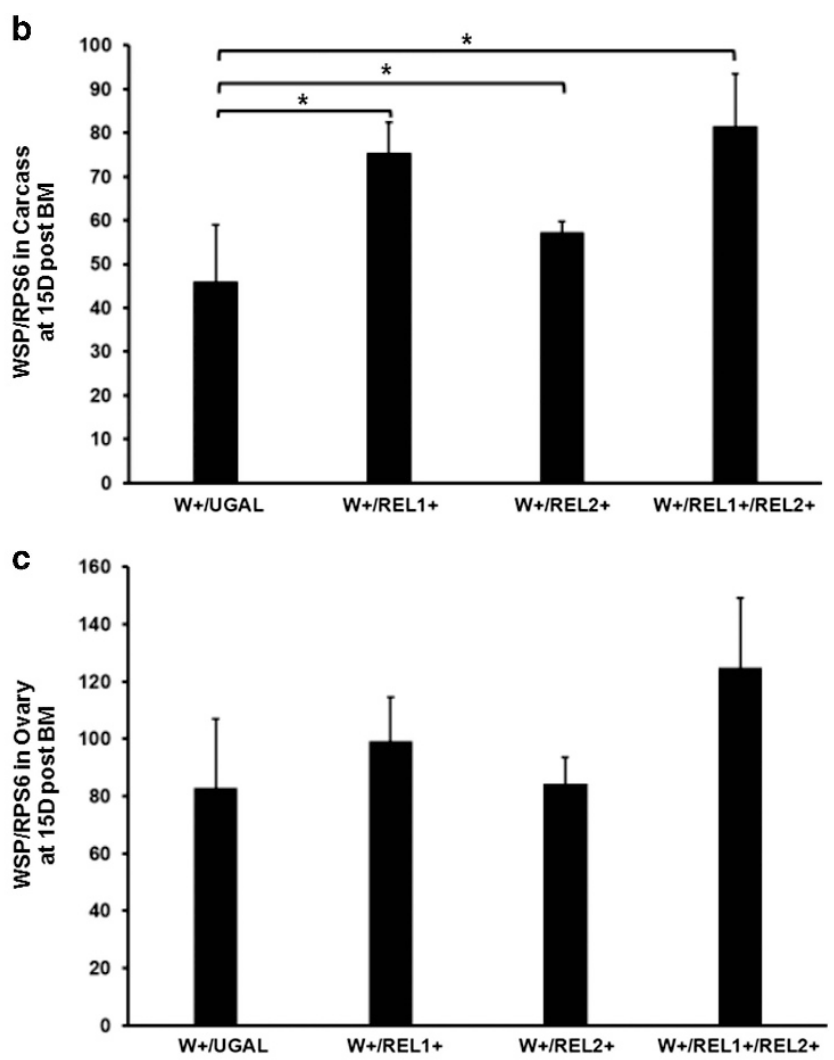

e

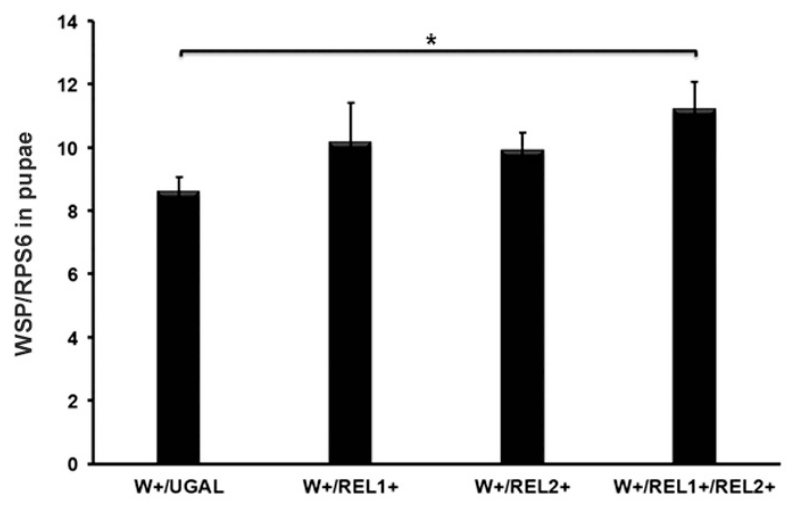

g

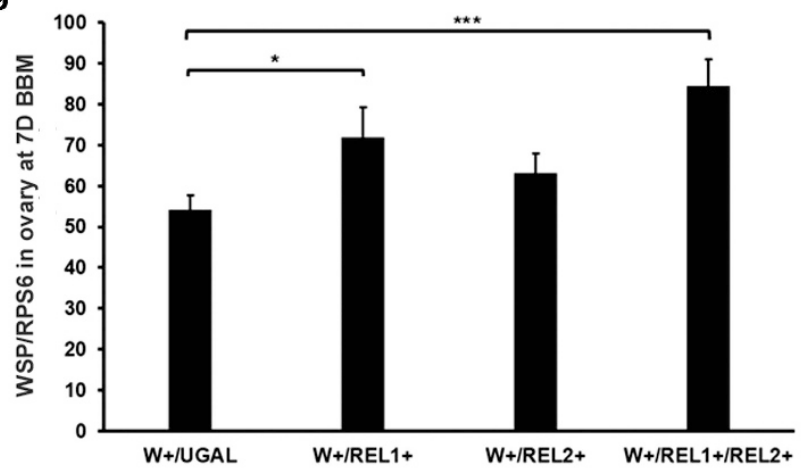


reports (Bian et al., 2005; Antonova et al., 2009), the expression of the transgenes did not change before a blood meal, but were induced markedly at $24 \mathrm{~h}$ post blood meal (Supplementary Figure S5), indicating that Wolbachia did not change the regulation patterns of transgene expression. We then compared wAlbB load in both the ovaries and the remaining carcass tissue of these mosquitoes at day 15 post blood meal. Wolbachia load was significantly higher in the carcasses of $\mathrm{W}+/$ REL1+, W+/REL2+ and W +/REL1+/REL2+ mosquitoes than in W+/UGAL mosquitoes (Figure 3b). No similar increase was observed in the ovaries (Figure 3c). Taken together, these results show that boosting immunity promotes proliferation of wAlbB in A. aegypti.

Maternal transmission of elevated Wolbachia densities in A. aegypti mosquitoes with enhanced immunity background

To test whether the elevated wAlbB infection could be maternally transmitted to the offspring of A. aegypti mosquitoes with a transgenically enhanced immunity background, we measured the wAlbB load in these offspring at the third instar larval and pupal stages. Higher wAlbB load was observed in both $\mathrm{W}+/$ REL2+ larvae $(P<0.01)$ and two immature stages of the W+/REL1+/REL2+
$(P<0.05$ for the larvae and $P<0.01$ for the pupae) transgenic mosquitoes than in W+/UGAL mosquitoes (Figures 3d and e). We further compared the wAlbB load at 7 days post eclosion in adult offspring that had not been blood fed and observed a significantly higher load of wAlbB in the carcasses of all three transgenic lines-W+/REL1+, W+/REL2+, and W+/REL1+/REL2+-than in the W+/UGAL line $(P<0.01)$ (Figure $3 \mathrm{f})$. A significant increase in $w$ AlbB load was also observed in the ovaries of non-blood fed W+/REL1+ and W+/REL1+/REL2+ mosquitoes when compared with W+/UGAL mosquitoes (Figure 3g). These results indicate that an elevated level of $w$ AlbB infection is maintained through maternal transmission of the bacteria to the offspring of transgenic $A$. aegypti mosquitoes.

Antioxidants, as potential effector molecules of boosted immunity, enhance Wolbachia infection in A. aegypti Overexpression of antimicrobial peptides was reported to increase tolerance to oxidant stress in Drosophila melanogaster (Zhao et al., 2011), and both $w \mathrm{AlbB}$ and boosting mosquito immunity were observed to induce the expression of antioxidants and antimicrobial peptides in A. aegypti (Pan et al., 2012). We speculated that boosting immunity enhances Wolbachia infection through alterations
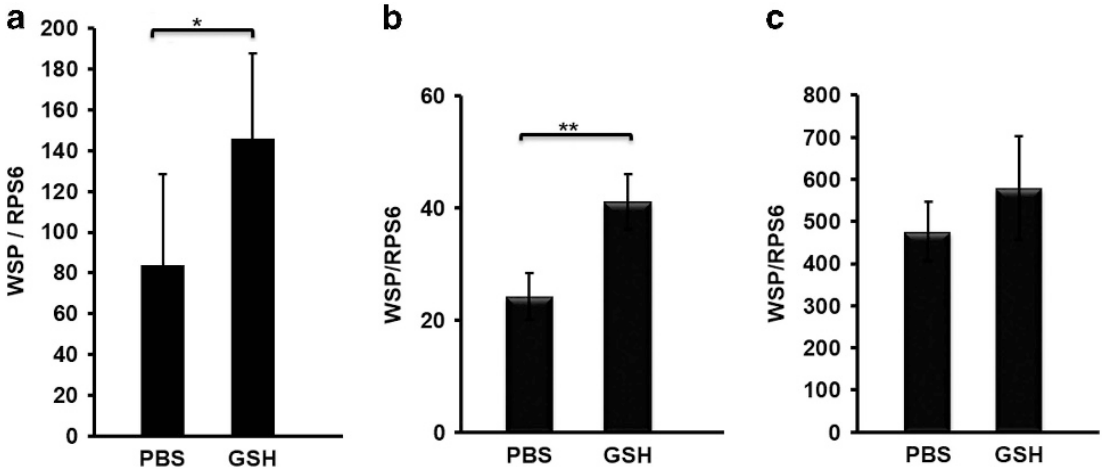

Figure 4 The impact of antioxidant treatment on Wolbachia abundance in transfected A. aegypti lines. Mosquitoes carrying wAlbB (a), wMel (b) and wMelPop (c) Wolbachia were injected with reduced L-glutathione (GSH) or PBS (control). The genome copy of Wolbachia was measured using q-PCR in the mosquito whole body 12 days post treatment. Each treatment had eight biological replicates with one mosquito each. Error bars indicate the standard error. Asterisks indicate significant difference of Wolbachia density in the treatment group compared with the control group. ${ }^{*} P<0.05 ;{ }^{*} P<0.01$; Mann-Whitney $U$-test.

Figure 3 Boosted basal immunity promotes the growth of wAlbB in A. aegypti. An introgression strategy was used to generate wAlbB infection in genetically modified A. aegypti with REL1, REL2 or both overexpressed (a). wAlbB infection is indicated by shading of the symbols: gray-filled with 'W+' represents wAlbB infection from the WB1 mosquito line; unshaded with 'T' represents transgenic $A$. aegypti mosquitoes (REL1+ or REL2+ line). Although the Wolbachia infection is maternally inherited, the genetic background is inherited from both parents. The theoretical percentage of transgenic mosquito genetic background is shown below the symbols. Repeated crosses of Wolbachia-infected females with transgenic males results in the Wolbachia-infected transgenic mosquito lines, W+/REL1+ and W+/REL2 + + W+/Ugal was generated as a control by crossing WB1 females with the wild-type Ugal strain in parallel. The wAlbB-infected hybrid line, $\mathrm{W}+/$ REL1+/REL2+, was generated based on a single cross of $\mathrm{W}+/ \mathrm{REL} 1+\left(\mathrm{G}_{6}\right)$ female and REL2+ male mosquitoes. The copy number of wAlbB in the carcass tissues (b) and ovaries (c) of the three transgenic mosquito lines (W+/REL1+, W+/REL2+ and W+/REL1+/REL2+) was compared with the W+/UGAL control group at day 15 post-blood feeding (PBM) using real-time PCR. The amount of wAlbB in the third instar larvae (d), pupae (e) and adults (f and $\mathbf{g}$ ) of the three transgenic lines was further measured using q-PCR and compared with the control group (W+/UGAL). For the adults, the Wolbachia density was measured in carcasses (f) and ovaries (g) of females at 7 days before blood meal (BBM). Each group contained eight biological replicates, each with an individual tissue. Error bars indicate standard error. Asterisks indicate significant difference of Wolbachia density in the transgenic lines compared with the control group. ${ }^{*} P<0.05$; ${ }^{*} P<0.01 ;{ }^{* *} P<0.001$; one-way ANOVA. 
in the redox balance. To explore whether an antioxidant could act as an effector molecule to regulate $w A$ AlbB infection levels, we injected WB1 mosquitoes intrathoracically with glutathione and then measured the wAlbB levels in mosquito whole bodies 12 days post treatment. We observed a 1.7fold increase in the wAlbB load in WB1 mosquitoes treated with glutathione compared with those treated with PBS as control $(P<0.05$; Figure $4 \mathrm{a})$. To test whether this regulation occurred across different Wolbachia strains in A. aegypti, we also did a similar comparison using $A$. aegypti carrying a stable $w \mathrm{Mel}$ (MGYP2 line) and wMelPop (PGYP1 line) (McMeniman et al., 2009; Walker et al., 2011). The glutathione treatment again caused a 1.7-fold increase in the amount of $w \mathrm{Mel}$ in the MGYP2 mosquito compared with control $(P<0.01)$ (Figure 4b). Although there was an increase in the wMelPop titer in the PGYP1 line relative to control, the difference was not statistically significant (Figure 4c). These results indicate that antioxidants may serve as the downstream effector molecules that enhance Wolbachia infection owing to their ability to counterbalance reactive oxygen species induced by this bacterium.

\section{Discussion}

Activation of the host's innate immune system by Wolbachia has been suggested as one mechanism causing pathogen interference in both naturally infected Drosophila and transinfected A. aegypti mosquitoes (Moreira et al., 2009; Bian et al., 2010; Rances et al., 2012; Wong et al., 2015), but the impact on Wolbachia itself has remained unclear. In this work, we have shown that both the IMD and Toll pathways in $A$. aegypti are triggered by introduction of the bacterium Wolbachia wAlbB. Enhancing these pathways causes an increase in the wAlbB titer, whereas silencing results in a reduction. Moreover, these elevated wAlbB infection levels are maintained through maternal transmission. We have also shown that antioxidants might serve as downstream effector molecules of the host immune system that enhance Wolbachia infection. Thus, our study has revealed the basis of interactions between Wolbachia and a newly acquired host.

Our results suggest that wAlbB can be sensed by the mosquito's innate immune system via PGRP-LE as a PRR, resulting in activation of the IMD pathway. This is consistent with the observation that PGRP-LE is an intracellular sensor of Gram-negative bacteria that induces the IMD pathway in Drosophila (Kaneko et al., 2006). It would be interesting to know whether PGRP-LE detects Wolbachia through its DAP-type peptidoglycan. Remarkably, boosted immunity markedly induces the expression of effector molecules that do not inhibit, but rather promote the proliferation of wAlbB in A. aegypti. This is most likely due to a lack of any specific targets for those antimicrobial peptides in the Wolbachia cell membrane. An increase in Wolbachia density was also reported in tsetse flies following induction of antimicrobial peptides by trypanosome infection (Rio et al., 2006). We think that an increase in Wolbachia density following immune boosting could be due to production of effector molecules that facilitate Wolbachia growth. One example could be antioxidants, the expression of which was previously shown to be induced or suppressed by activation or silencing, respectively, of the Toll pathway in A. aegypti (Pan et al., 2012). In Drosophila, overexpression of antimicrobial peptides increases antioxidant enzyme activities and changes the cellular redox balance, facilitating fly survival in hyperoxia (Zhao et al., 2011). Consistent with our observation in both WB1 and MGYP2 mosquitoes, previous studies also showed that antioxidant treatment resulted in increased Wolbachia density in Drosophila (Brennan et al., 2012). It is also possible that these induced antimicrobial peptides provide an indirect benefit to Wolbachia by removing the susceptible microbial flora from within mosquitoes and generating new niches. As a consequence, Wolbachia may utilize the immune boost to exclude its competitors and take their place in various tissues. Taken together, Wolbachia activates mosquito immune pathways, which leads to promotion of its own growth, indicating that there is a positive feedback loop between the host immune system and Wolbachia load. In transinfected A. aegypti lines, the observed immune system boost provides survival signals for successful establishment of a novel Wolbachia symbiosis.

Previous studies have also shown that the host immune system interacts with symbiotic bacteria to foster relationships in other systems. In D. melanogaster, the endosymbiont Spiroplasma is not susceptible to either the cellular or humoral arms of the host immune system, and activation of the Toll and IMD immune pathways results in an increase in the Spiroplasma titer (Herren and Lemaitre, 2011). Evidence from both invertebrate and vertebrate models reveals that innate immune receptors are required to promote long-term colonization by the microbiota (Chu and Mazmanian, 2013). The host immune system has a central role in shaping the composition of the microbiota as well as its proximity to host tissues (Hooper et al., 2012). The immune system of organisms ranging from hydra to mammals can distinguish beneficial from harmful bacteria via PRRs and interact with symbionts in a way that promotes the establishment and maintenance of beneficial symbioses (Nyholm and Graf, 2012). Alternatively, symbiotic bacteria may utilize the host immune system to actively promote highly evolved associations with their hosts (Hooper et al., 2012; Nyholm and Graf, 2012; Chu and Mazmanian, 2013). Wolbachia may use a similar strategy to exploit the host immunity to establish symbiosis. 
Boosting of both the Toll and IMD pathways was consistent with the function assay through comparison of the survivorship of wAlbB-infected and uninfected $A$. aegypti after challenge by Gram-negative bacteria, Gram-positive bacteria and fungi. We observed that wAlbB protects WB1 mosquitoes from all three types of pathogen. This is in agreement with a previous report showing that both $w \mathrm{Mel}$ and $w \mathrm{MelPop}$ can protect $A$. aegypti from infection with Gram-negative bacteria (Erwinia carotovora and Salmonella typhimurium), and wMelPop can increase survival of mosquitoes after challenge with Gram-positive bacteria (Mycobacterium marinum) (Ye et al., 2013). Thus, Wolbachiaboosted immunity is beneficial to the mosquito host owing to Wolbachia-associated protection against pathogens. In turn, this facilitates establishment of the long-term relationship between Wolbachia and its new mosquito host. As defensive symbionts, Spiroplasma and Hamiltonella defensa protect their hosts from attack by a nematode and a parasitoid, respectively, promoting spread of symbionts in natural populations (Oliver et al., 2008; Jaenike et al., 2010). Wolbachia might use a similar strategy to accelerate and maintain its cytoplasmic incompatibility-driven spread, facilitating the current effort to develop a Wolbachia-based population replacement for dengue and Zika control.

The work reported here has three important implications. First, mosquito innate immunity can be finely adjusted such that it inhibits pathogens but promotes endosymbionts. Understanding this mechanism will greatly facilitate the development of endosymbionts as a tool for blocking pathogen transmission. For example, boosting immunity through mosquito transgenesis could be used to enhance both pathogen refractoriness and Wolbachia symbiosis simultaneously. Second, the interplay between the mosquito innate immune system and symbiosis can occur in two directions. On the one hand, the mosquito microbiota facilitates the buildup of basal immunity, as removal of the microbiota reduces the level of basal immunity (Xi et al., 2008b; Dong et al., 2009). On the other hand, a high level of basal immunity facilitates the formation of symbioses and promotes the growth of the specific microbes within the mosquito. Finally, the effector molecules induced by mosquito immune pathways, including Wolbachia-upregulated antimicrobial peptides such as DEF and CEC, do not inhibit the growth of Wolbachia. Future research should investigate how innate immunity can be utilized to shape the microbiota, with regard to its structure, distribution and amount, such that the mosquito physiological environment can be modified to become inhospitable to human pathogens. This could lead to development of novel strategies for blocking the transmission of human pathogens through mosquito vectors.

In addition to the Toll and IMD pathways, it is also worthwhile to explore the role of the JAK/STATB (Janus Kinase-Signal Transducer Activator of
Transcription) and JNK (c-Jun N-terminal kinase) pathways in the Wolbachia-host symbiotic relationship in the future studies. Induction of the JAK/ STAT pathway by $w \mathrm{Mel}$ has been reported in $A$. aegypti (Terradas et al., 2017). Evidence also shows that the JAK/STAT pathway controls DENV infection in A. aegypti (Souza-Neto et al., 2009) and West Nile virus infection in Culex mosquitoes (Paradkar et al., 2012). Previous studies also show that Wolbachia upregulates genes in the JNK pathway (Xi et al., 2008a; Kremer et al., 2012), and this pathway was reported to confer tolerance to oxidative stress and extend life span in Drosophila (Wang et al., 2003). Thus, Wolbachia might utilize the host JNK pathway to facilitate its growth through regulation of host oxidative metabolism. It will be interesting to further study how cross-talk between host immune and metabolic pathways facilitates the Wolbachia-host communication to establish symbiosis.

Finally, the present study leads to deeper understanding of the evolution of symbiosis and pathogen blocking. We show that Wolbachia exploits the host immune system to establish a symbiotic relationship with its new mosquito host. Previous studies have also found that the symbiotic bacterium Sodalis induces a strong expression of immunity-related genes that is essential for its ability to persistently infect tsetse flies (Weiss et al., 2008). Boosted immunity promotes proliferation of Wolbachia, but inhibits both mosquito and human pathogens. It appears that long-term adaptations between Wolbachia and its host may lead to a waning of the Wolbachia-induced immune response, resulting in reduction of pathogen blocking and attenuation of Wolbachia titer, as observed in $A$. albopictus. It will be worth testing whether loss of Wolbachia infection can occur from a break in the positive feedback loop among Wolbachia, host immunity, and antioxidants. Our results show that Wolbachia in somatic tissue (carcasses) may be more sensitive to immune regulation than in ovaries, indicating that attenuation of pathogen blocking may be more likely to occur than loss of symbiosis. Further understanding of the interplay between Wolbachia and host immunity will facilitate a long-term projection of the stability of the Wolbachia-A. aegypti mosquito system that is being used in the control of Aedes mosquitoes and dengue.

\section{Materials and methods}

DNA and RNA extraction, PCR, quantitative reversetranscription polymerase chain reaction, RNAi, IFA, microbial challenge and survival assays and antioxidant treatment were conducted using standard methods, details of which are available in the supplementary materials and methods.

Mosquito rearing and cell culture

Waco is a Wolbachia-free, wild-type A. aegypti mosquito line. WB1 (Xi et al., 2005), wMel (Walker et al., 2011) and wMelPop-CLA (McMeniman et al., 
2009) are A. aegypti mosquito lines that stably carry Wolbachia wAlbB, wMel or wMelPop infections, respectively. Prior to use, the WB1 line was out-crossed with the Waco line for $>10$ generations to ensure a similar genomic background. WBT is an A. aegypti mosquito line, in which the wAlbB infection was cleared by tetracycline treatment of WB1, and has subsequently been maintained for $>10$ generations. Vg- $\Delta$ REL1-A (REL1+) and Vg-REL2 (REL2+) are transgenic $A$. aegypti mosquito lines, in which the Rel1 and Rel2 genes, respectively, are overexpressed (Bian et al., 2005; Antonova et al., 2009). The Rockefeller/UGAL is a wild-type A. aegypti line used as a control for the transgenic lines. All mosquito lines used in this study were maintained under standard laboratory conditions of $28^{\circ} \mathrm{C}$ and $80 \%$ humidity, with a $12-\mathrm{h} / 12-\mathrm{h}$ light/ dark cycle, as previously reported (Pan et al., 2012). This study was carried out in strict accordance with the recommendations set out in the Guide for the Care and Use of Laboratory Animals of the National Institutes of Health. The protocol was approved by Michigan State University Animal Care and Use Committees.

The wAlbB-infected W-Aag2 cell line was maintained in Schneider's Drosophila cell culture media supplemented with L-glutamine, $10 \%$ fetal bovine serum (v/v) and 1\% penicillin/streptomycin (Life Technologies, Carlsbad, CA, USA) at $26^{\circ} \mathrm{C}$ following standard procedures described previously (Lu et al., 2012).

Introduction of Wolbachia into transgenic mosquitoes An introgression strategy was used to generate wAlbB-infected A. aegypti lines overexpressing REL1(REL1+), REL2 (REL2+) or both (REL1 +/REL2+) (Brelsfoard et al., 2008). Transgenic mosquitoes identified by the EGFP eye marker were chosen for this study. In total, 50 WB1 virgin females were crossed with 50 virgin males of each transgenic $A$. aegypti mosquito line REL1+. REL2+ as well as the wild-type Rockefeller/UGAL at 12 days post eclosion. The outcross was repeated for seven generations, so that the lines shared over $99 \%$ genomic identity with their transgenic counterparts. A hybrid line (W+/REL1+/REL2+) was established through a single outcross between 50 wAlbB-infected Rel1+ (W+/REL1+) virgin females (G6) and 50 Rel2+ virgin males. The wAlbB infection was stably maintained in the $W+/$ REL1 + , W+/REL2+, W+/REL1+/REL2+ and W+/ Ugal mosquito lines throughout the course of the experiment.

\section{Conflict of Interest}

Dr $\mathrm{Xi}$ is also affiliated to Guangzhou Wolbaki Biotech Co., LTD. The remaining authors declare no conflict of interest.

\section{Acknowledgements}

We thank Dr Todd A Ciche for providing E. cloacae and M. luteus, and Professor Scott O'Neill for providing MGYP2 and PGYP1 lines. Funding information: this work was supported by the National Institutes of Health/ National Institute of Allergy and Infectious Disease R01AI080597, the United States Agency for International Development (USAID) Combating Zika and Future Threats program, and Guangdong Innovative Research Team Program (No. 2011S009). The funders had no role in study design, data collection and interpretation, or the decision to submit the work for publication.

\section{References}

Antonova Y, Alvarez KS, Kim YJ, Kokoza V, Raikhel AS. (2009). The role of NF-kappaB factor REL2 in the Aedes aegypti immune response. Insect Biochem Mol Biol 39: 303-314.

Bhatt S, Gething PW, Brady OJ, Messina JP, Farlow AW, Moyes CL et al. (2013). The global distribution and burden of dengue. Nature 496: 504-507.

Bian G, Shin SW, Cheon HM, Kokoza V, Raikhel AS. (2005). Transgenic alteration of Toll immune pathway in the female mosquito Aedes aegypti. Proc Natl Acad Sci USA 102: 13568-13573.

Bian G, Xu Y, Lu P, Xie Y, Xi Z. (2010). The endosymbiotic bacterium Wolbachia induces resistance to dengue virus in Aedes aegypti. PLoS Pathog 6: e1000833.

Bian G, Joshi D, Dong Y, Lu P, Zhou G, Pan X et al. (2013). Wolbachia invades Anopheles stephensi populations and induces refractoriness to Plasmodium infection. Science 340: 748-751.

Bourtzis K, Pettigrew MM, O'Neill SL. (2000). Wolbachia neither induces nor suppresses transcripts encoding antimicrobial peptides. Insect Mol Biol 9: $635-639$.

Bourtzis K, Dobson SL, Xi Z, Rasgon JL, Calvitti M, Moreira LA et al. (2014). Harnessing mosquitoWolbachia symbiosis for vector and disease control. Acta Trop 132: S150-S163.

Brelsfoard CL, Sechan Y, Dobson SL. (2008). Interspecific hybridization yields strategy for South Pacific filariasis vector elimination. PLoS Negl Trop Dis 2: e129.

Brennan LJ, Haukedal JA, Earle JC, Keddie B, Harris HL. (2012). Disruption of redox homeostasis leads to oxidative DNA damage in spermatocytes of Wolbachia-infected Drosophila simulans. Insect Mol Biol 21: 510-520.

Chu H, Mazmanian SK. (2013). Innate immune recognition of the microbiota promotes host-microbial symbiosis. Nat Immunol 14: 668-675.

Dimopoulos G. (2003). Insect immunity and its implication in mosquito-malaria interactions. Cell Microbiol 5: $3-14$.

Dobson SL. (2003). Reversing Wolbachia-based population replacement. Trends Parasitol 19: 128-133.

Dong Y, Aguilar R, Xi Z, Warr E, Mongin E, Dimopoulos G. (2006). Anopheles gambiae immune responses to human and rodent Plasmodium parasite species. PLoS Pathog 2: e52. 
Dong Y, Manfredini F, Dimopoulos G. (2009). Implication of the mosquito midgut microbiota in the defense against malaria parasites. PLoS Pathog 5: e1000423.

Dutra HL, Rocha MN, Dias FB, Mansur SB, Caragata EP, Moreira LA. (2016). Wolbachia blocks currently circulating Zika virus isolates in Brazilian Aedes aegypti mosquitoes. Cell Host Microbe 19: 771-774.

Frolet C, Thoma M, Blandin S, Hoffmann JA, Levashina EA. (2006). Boosting NF-kappaB-dependent basal immunity of Anopheles gambiae aborts development of Plasmodium berghei. Immunity 25: 677-685.

Herren JK, Lemaitre B. (2011). Spiroplasma and host immunity: activation of humoral immune responses increases endosymbiont load and susceptibility to certain Gram-negative bacterial pathogens in Drosophila melanogaster. Cell Microbiol 13: 1385-1396.

Hoffmann AA, Montgomery BL, Popovici J, IturbeOrmaetxe I, Johnson PH, Muzzi F et al. (2011). Successful establishment of Wolbachia in Aedes populations to suppress dengue transmission. Nature 476: $454-457$.

Hooper LV, Littman DR, Macpherson AJ. (2012). Interactions between the microbiota and the immune system. Science 336: 1268-1273.

Jaenike J, Unckless R, Cockburn SN, Boelio LM, Perlman SJ. (2010). Adaptation via symbiosis: recent spread of a Drosophila defensive symbiont. Science 329: $212-215$.

Kambris Z, Cook PE, Phuc HK, Sinkins SP. (2009). Immune activation by life-shortening Wolbachia and reduced filarial competence in mosquitoes. Science 326: 134-136.

Kaneko T, Yano T, Aggarwal K, Lim JH, Ueda K, Oshima Y et al. (2006). PGRP-LC and PGRP-LE have essential yet distinct functions in the drosophila immune response to monomeric DAP-type peptidoglycan. Nat Immunol 7: 715-723.

Kittayapong P, Baisley KJ, Baimai V, O'Neill SL. (2000). Distribution and diversity of Wolbachia infections in Southeast Asian mosquitoes (Diptera: Culicidae). J Med Entomol 37: 340-345.

Kremer N, Charif D, Henri H, Gavory F, Wincker P, Mavingui $P$ et al. (2012). Influence of Wolbachia on host gene expression in an obligatory symbiosis. BMC Microbiol 12: S7.

Kumar S, Molina-Cruz A, Gupta L, Rodrigues J, BarillasMury C. (2010). A peroxidase/dual oxidase system modulates midgut epithelial immunity in Anopheles gambiae. Science 327: 1644-1648.

Lu P, Bian G, Pan X, Xi Z. (2012). Wolbachia induces density-dependent inhibition to dengue virus in mosquito cells. PLoS Negl Trop Dis 6: e1754.

McMeniman CJ, Lane RV, Cass BN, Fong AW, Sidhu M, Wang YF et al. (2009). Stable introduction of a lifeshortening Wolbachia infection into the mosquito Aedes aegypti. Science 323: 141-144.

McMeniman CJ, O'Neill SL. (2010). A virulent Wolbachia infection decreases the viability of the dengue vector Aedes aegypti during periods of embryonic quiescence. PLoS Negl Trop Dis 4: e748.

Meister S, Kanzok SM, Zheng XL, Luna C, Li TR, Hoa NT et al. (2005). Immune signaling pathways regulating bacterial and malaria parasite infection of the mosquito Anopheles gambiae. Proc Natl Acad Sci USA 102: 11420-11425.

Molloy JC, Sinkins SP. (2015). Wolbachia do not induce reactive oxygen species-dependent immune pathway activation in aedes albopictus. Viruses 7: 4624-4639.

Moreira LA, Iturbe-Ormaetxe I, Jeffery JA, Lu G, Pyke AT, Hedges LM et al. (2009). A Wolbachia symbiont in Aedes aegypti limits infection with dengue, Chikungunya, and Plasmodium. Cell 139: 1268-1278.

Nyholm SV, Graf J. (2012). Knowing your friends: invertebrate innate immunity fosters beneficial bacterial symbioses. Nat Rev Microbiol 10: 815-827.

Oliver KM, Campos J, Moran NA, Hunter MS. (2008). Population dynamics of defensive symbionts in aphids. Proc Biol Sci 275: 293-299.

Pan X, Zhou G, Wu J, Bian G, Lu P, Raikhel AS et al. (2012). Wolbachia induces reactive oxygen species (ROS)-dependent activation of the Toll pathway to control dengue virus in the mosquito Aedes aegypti. Proc Natl Acad Sci USA 109: E23-E31.

Paradkar PN, Trinidad L, Voysey R, Duchemin JB, Walker PJ. (2012). Secreted Vago restricts West Nile virus infection in Culex mosquito cells by activating the JakSTAT pathway. Proc Natl Acad Sci USA 109: 18915-18920.

Rances E, Ye YH, Woolfit M, McGraw EA, O'Neill SL. (2012). The relative importance of innate immune priming in Wolbachia-mediated dengue interference. PLoS Pathog 8: e1002548.

Ricci I, Cancrini G, Gabrielli S, D'Amelio S, Favi G. (2002). Searching for Wolbachia (Rickettsiales: Rickettsiaceae) in mosquitoes (Diptera: Culicidae): large polymerase chain reaction survey and new identifications. $J$ Med Entomol 39: 562-567.

Rio RV, Wu YN, Filardo G, Aksoy S. (2006). Dynamics of multiple symbiont density regulation during host development: tsetse fly and its microbial flora. Proc Biol Sci 273: 805-814.

Shin SW, Kokoza V, Bian G, Cheon HM, Kim YJ, Raikhel AS. (2005). REL1, a homologue of Drosophila dorsal, regulates toll antifungal immune pathway in the female mosquito Aedes aegypti. J Biol Chem 280: 16499-16507.

Souza-Neto JA, Sim S, Dimopoulos G. (2009). An evolutionary conserved function of the JAK-STAT pathway in anti-dengue defense. Proc Natl Acad Sci USA 106: 17841-17846.

Terradas G, Joubert DA, McGraw EA. (2017). The RNAi pathway plays a small part in Wolbachia-mediated blocking of dengue virus in mosquito cells. Sci Rep 7: 43847.

Walker T, Johnson PH, Moreira LA, Iturbe-Ormaetxe I, Frentiu FD, McMeniman CJ et al. (2011). The wMel Wolbachia strain blocks dengue and invades caged Aedes aegypti populations. Nature 476: 450453.

Wang MC, Bohmann D, Jasper H. (2003). JNK signaling confers tolerance to oxidative stress and extends lifespan in Drosophila. Dev Cell 5: 811-816.

Waterhouse RM, Kriventseva EV, Meister S, Xi Z, Alvarez KS, Bartholomay LC et al. (2007). Evolutionary dynamics of immune-related genes and pathways in disease-vector mosquitoes. Science 316: 1738-1743.

Weiss BL, Wu Y, Schwank JJ, Tolwinski NS, Aksoy S. (2008). An insect symbiosis is influenced by bacterium-specific polymorphisms in outermembrane protein A. Proc Natl Acad Sci USA 105: 15088-15093. 
Werren JH, Baldo L, Clark ME. (2008). Wolbachia: master manipulators of invertebrate biology. Nat Rev Microbiol 6: 741-751.

Wong ZS, Brownlie JC, Johnson KN. (2015). Oxidative stress correlates with wolbachia-mediated antiviral protection in wolbachia-Drosophila associations. Appl Environ Microbiol 81: 3001-3005.

Xi Z, Khoo CC, Dobson SL. (2005). Wolbachia establishment and invasion in an Aedes aegypti laboratory population. Science 310: 326-328.

Xi Z, Gavotte L, Xie Y, Dobson SL. (2008a). Genome-wide analysis of the interaction between the endosymbiotic bacterium Wolbachia and its Drosophila host. BMC Genomics 9: 1.

Xi Z, Ramirez JL, Dimopoulos G. (2008b). The Aedes aegypti toll pathway controls dengue virus infection. PLoS Pathog 4: e1000098.

Yano T, Mita S, Ohmori H, Oshima Y, Fujimoto Y, Ueda R et al. (2008). Autophagic control of listeria through intracellular innate immune recognition in drosophila. Nat Immunol 9: 908-916.

Ye YH, Woolfit M, Rances E, O'Neill SL, McGraw EA. (2013). Wolbachia-associated bacterial protection in the mosquito Aedes aegypti. PLoS Negl Trop Dis 7: e2362.
Zhao HW, Zhou D, Haddad GG. (2011). Antimicrobial peptides increase tolerance to oxidant stress in Drosophila melanogaster. $J$ Biol Chem 286: 6211-6218.

Zou Z, Souza-Neto J, Xi Z, Kokoza V, Shin SW, Dimopoulos G et al. (2011). Transcriptome analysis of Aedes aegypti transgenic mosquitoes with altered immunity. PLoS Pathog 7: e1002394.

(c) (i) This work is licensed under a Creative Commons Attribution 4.0 International License. The images or other third party material in this article are included in the article's Creative Commons license, unless indicated otherwise in the credit line; if the material is not included under the Creative Commons license, users will need to obtain permission from the license holder to reproduce the material. To view a copy of this license, visit http:// creativecommons.org/licenses/by/4.0/

(c) The Author(s) 2018

Supplementary Information accompanies this paper on The ISME Journal website (http://www.nature.com/ismej) 